\title{
Application Of The Interlaced Sweep Method For The Solution Of Problems In Field Theory
}

\author{
Aloizs Ratnieks, Marina Uhanova \\ Riga Technical University, Faculty of Computer science and Information technology. \\ Address: 1/4 Meza str., Riga, LV-1048, Latvia
}

\begin{abstract}
For solution of problems in field theory the method of sweep is very popular. The authors suggest a very effective method of interlaced sweep. The essence of the interlaced sweep method lies in the fact that matrix of the linear algebraic equations system is broken into parts and the solution of separate blocks is sought by direct methods. Usually for each line of the grid a separate block is created. The system of block equations has a tridiagonal matrix where only elements of the main diagonal and two neighboring diagonals are different from zero. The system of equations with such a matrix is easily solved by the method of elimination of unknowns.

By solving the problems by the finite element method, the nodes of touching of neighboring elements can be placed on curved lines, and the sweep on these lines can be performed observing the principle of interlaced sweep. By following this method, the neighboring lines should not belong to the same half-step.
\end{abstract}

Keywords: sweep method, differential equation, system of equations.

\section{INTRODUCTION}

The solution of wide range of practically important problems can be reduced to calculation of various fields which are described by differential equations in partial derivatives [1]. By application of the finite difference method or of the finite element method, the solution of differential equation in partial derivatives is reduced to solution of system of equations. The matrix of this system usually has very high order where only small proportion of coefficients differs from zero [2].

The solution of this system by the method of elimination of unknown according to Gauss scheme is inefficient because it leads to increase of number of unequal to zero coefficients in the matrix.

For solution of difference equations problems in field theory the method of sweep is very popular. There are many different modifications of this method. The speed of convergence of different sweep method modifications is influenced by relative value of the matrix's diagonal elements relating to the previous step, to alternation of sweep directions, to type of displacement used, to periodic change of displacement for reverse and other factors [3]. The authors suggest a very effective method of interlaced sweep which is neither a method of simultaneous displacements, nor a method of successive displacements along the lines.

\section{DESCRIPTION OF THE INTERLACED SWEEP METHOD}

The essence of the sweep method lies in the fact that matrix of the linear algebraic equations system received as a result of the finite difference approximation of differential equation in partial derivatives, is broken into parts and the solution of separate blocks is sought by direct methods. Moreover, the equations included in the block are met exactly. Usually for each line of the grid a separate block is created. The equations system of block has a tridiagonal matrix where only elements of the main diagonal and two neighboring diagonals are different from zero. All other elements of this matrix are equal to zero. The system of equations with such a matrix is usually solved by the method of elimination of unknowns.

In the case of interlace sweep application in twodimensional area, each iteration consists of two half steps which alternate:

- sweep on odd gridlines;

- sweep on even gridlines.

Let us denote:

$\mathrm{k}$ - number of grid line;

$\mathrm{n}$ - number of grid node on the line;

$\mathrm{U}_{\mathrm{kn}}$ - component of the required vector of the grid node with indices $\mathrm{k}$ and $\mathrm{n}$; 
$\mathrm{PX}_{\mathrm{kn}}$ - coefficient of the matrix corresponding to the grid node with indices $\mathrm{k}$ and $\mathrm{n}$ of the line directed along $\mathrm{X}$ - axis;

$\mathrm{PY}_{\mathrm{kn}}$ - coefficient of the matrix corresponding to the grid node with indices $\mathrm{k}$ and $\mathrm{n}$ of the line directed along Y - axis;

$\mathrm{F}_{\mathrm{kn}}$ - right-hand member of the equation which corresponds to the grid node with indices $\mathrm{k}$ and $\mathrm{n}$;

$\mathrm{j}$ - serial number of iteration;

ni - current iteration number;

$\mathrm{kr}$ - relaxation coefficient;

$\mathrm{h}$ - step of change of grid nodes coordinates.

When solving problems for one-dimensional field by the sweep method, all diagonal elements of the matrix correspond entirely to the current step, i.e. relaxation coefficient is equal to 1 .

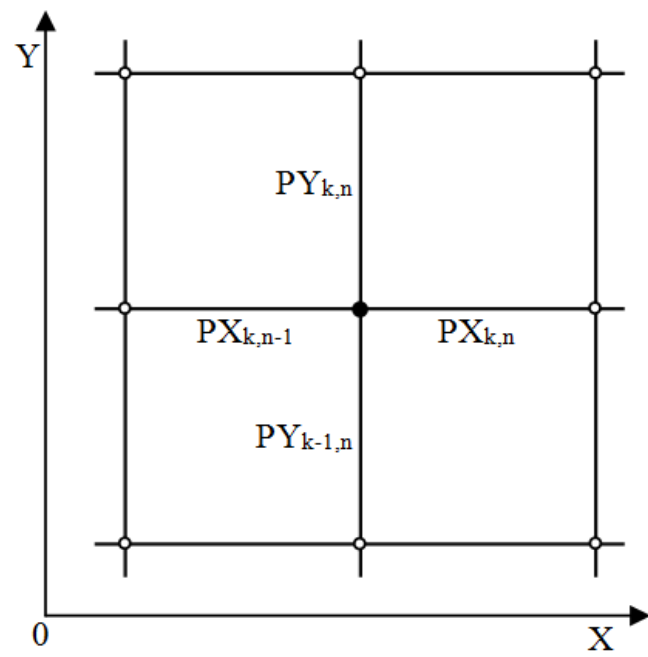

Fig. 1 Designations of grid elements.

To compensate partially the errors resulting from the transfer of some matrix coefficients of the initial equation system to the previous half-step and the increase of the convergence speed in solving the twodimensional and three-dimensional problems, the recurrence formulas of iterative process can be changed so that the components of the required vector are determined by a factor of relaxation directly inside the block. In other words, each component of the required vector would be calculated based on the assumption that all other components of the vector have been calculated with the same coefficient of relaxation.

To achieve this goal, the recurrence formula of the half-step of the iteration process for $\mathrm{n}$ - the grid node depicted in Fig. 1 and sweep along the grid line directed along the $\mathrm{x}$-axis must be written in the form (1).

$$
\begin{aligned}
& P X_{k, n-1} \cdot U_{k, n-1}^{j+1 / 2}-D X_{k n} \cdot U_{k n}^{j+1 / 2}+P X_{k n} \cdot U_{k, n+1}^{j+1 / 2}= \\
& =F_{k n}-P Y_{k-1, n} \cdot U_{k-1, n}^{j}-P Y_{k n} \cdot U_{k+1, n}^{j}+ \\
& +(1-1 / k r) \cdot\left(P X_{k, n-1}+P X_{k n}\right) \cdot U_{k n}^{j-1 / 2}
\end{aligned}
$$

where:

$$
D X_{k n}=\left(P X_{k, n-1}+P X_{k n}\right) / k r+P Y_{k-1, n}+P Y_{k n}
$$

From the above recurrence formula of the iterative process follows that the coefficients of the main diagonal of the matrix in one-dimensional area are substituted by corresponding coefficients of the main diagonal of the matrix for initial problem for two or three dimensional area. Therefore, only that part of diagonal elements should be divided be the coefficient of relaxation which corresponds to the diagonal elements of the matrix of one-dimensional area. In the case of a sweep along the grid line which is directed along the y-axis, the coefficients of the main diagonal of the matrix of one dimensional area are equal to the sum of coefficients $P Y_{k-1, n}$ and $P Y_{k, n}$ with the opposite sign.

One part of the diagonal element of the matrix of one-dimensional area, obtained as a result of division by the coefficient of relaxation refers to the current half-step, but the remaining part refers to previous iteration. If the coefficient of relaxation is equal to one then all diagonal elements of the matrix of the initial problem relate entirely to the current half-step.

\section{DESCRIPTION OF CONTROL EXAMPLES}

To evaluate the effectiveness of interlaced sweep method in solving two-dimensional problems the following equation (2) was being solved in the square area with dimensions $32 \times 32$, containing 900 internal grid nodes.

$\frac{\partial}{\partial x}\left(\sigma \frac{\partial U}{\partial x}\right)+\frac{\partial}{\partial y}\left(\sigma \frac{\partial U}{\partial y}\right)=f$

This equation has exact analytical solution (3):

$U(x, y)=x^{3} y^{3}$

by

$\sigma=\frac{1}{x y}, \quad f=3\left(x^{2}+y^{2}\right)$

The potentials in the boundary nodes of the grid must be calculated by the function $U(x, y)=x^{3} y^{3}$. To solve this equation the following program was written: 


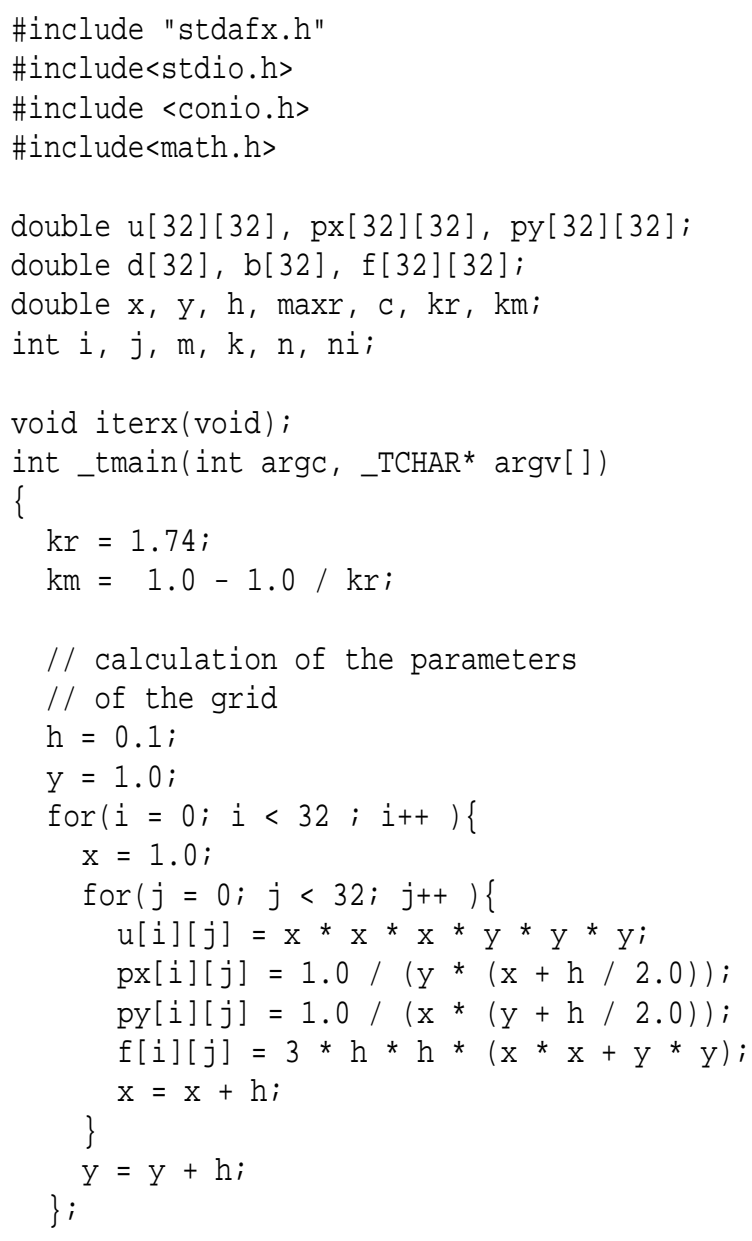

$/ /$ assign 0.0 to the potentials of $/ /$ all internal nodes of the grid

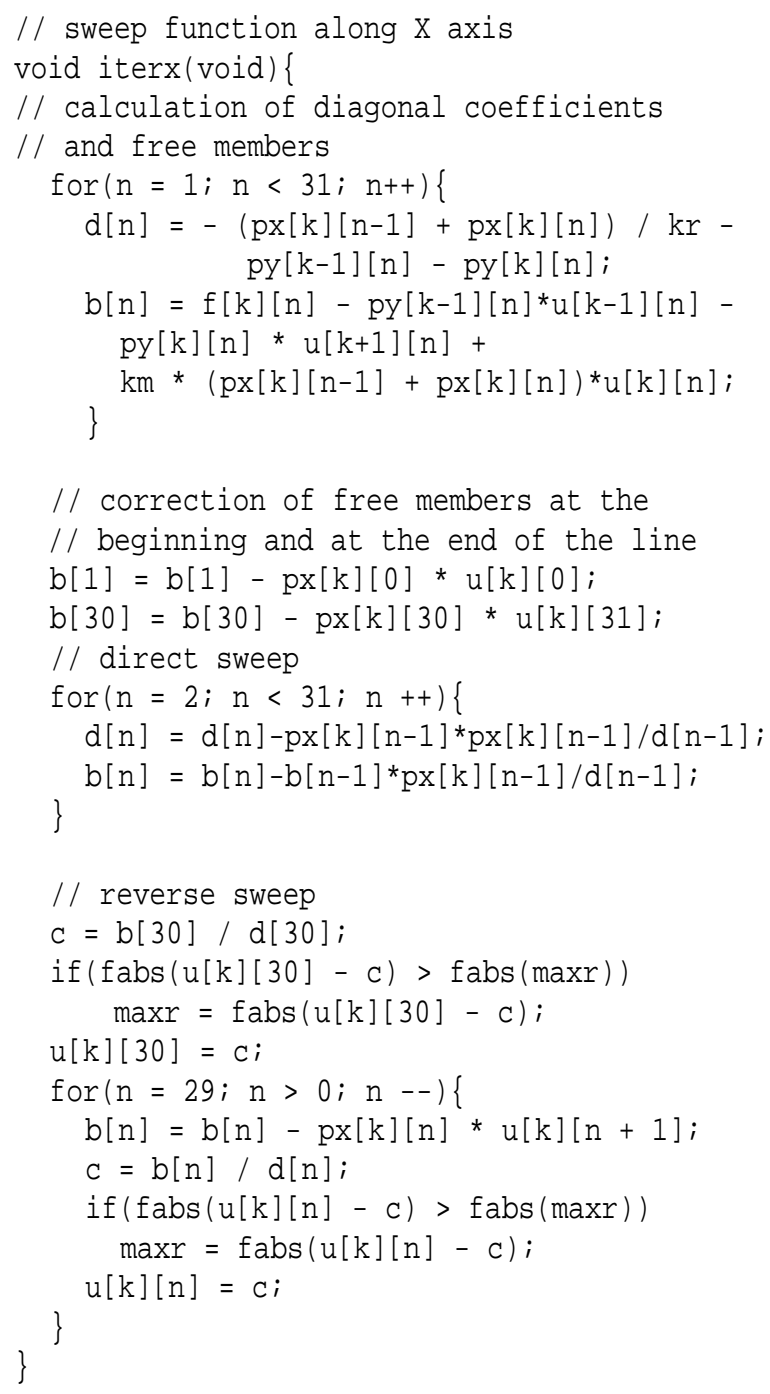

By solving the problem using the Microsoft Visual Studio Express Edition 2012, the absolute value of maximum correction to the solution has decreased in $10^{6}$ times in 47 iterations by $\mathrm{kr}=1.74$. The total error of the finite approximation and other factors in the middle point of the area with coordinates $(2.5,2.5)$ is equal to $0.016 \%$.

To evaluate the effectiveness of the interlaced sweep method in solving three-dimensional problems the following equation (5) was being solved in the area having the form of the cube with dimensions $32 \times 32 \times 32$, containing 27000 internal grid nodes.

$\frac{\partial}{\partial x}\left(\sigma \frac{\partial U}{\partial x}\right)+\frac{\partial}{\partial y}\left(\sigma \frac{\partial U}{\partial y}\right)+\frac{\partial}{\partial z}\left(\sigma \frac{\partial U}{\partial z}\right)=f$

This equation has an exact analytical solution (6):

$U(x, y, z)=x^{3} y^{3} z^{3}$

at 


$$
\sigma=\frac{1}{x y z}, \quad f=3\left(x^{2} y^{2}+x^{2} z^{2}+y^{2} z^{2}\right)
$$

The potentials in the boundary nodes of the grid must be calculated by the function $U(x, y, z)=x^{3} y^{3} z^{3}$.

To solve the problems for three-dimensional area the principle of interlaced sweep must be observed in all planes which have sweep lines.

When solving the problems the absolute value of maximum correction for solution is reduced in $10^{6}$ times in 47 iterations by $\mathrm{kr}=7.9$. The total error of the finite approximation and other factors in the midpoint of the field with coordinates $(2.5,2.5,2.5)$ were equal to $0.017 \%$.

Relatively large value of the coefficient of relaxation is explained by the fact that in threedimensional case just four coefficients of the initial system matrix relate to the previous half-step and only small part of the coefficient which belongs to the main matrix diagonal in the initial system is divided by the coefficient of relaxation.

To solve the problems in two and three dimensional fields, the authors decided to modify the sweep method for sequential displacement along the grid lines by means of substitution of recurrence formulas of the iteration process for the sweep method by replacement formulas for two and three dimensional area where only one part of the diagonal element corresponding to the matrix of the one-dimensional field is divided by the coefficient of relaxation. This modification of the sweep method has increased the speed of convergence of iteration process in two and three dimensional fields in several times.

The execution of iterations by solving the control example by all above mentioned methods was interrupted when the maximum module of correction to the solution decreased in $10^{6}$ times.

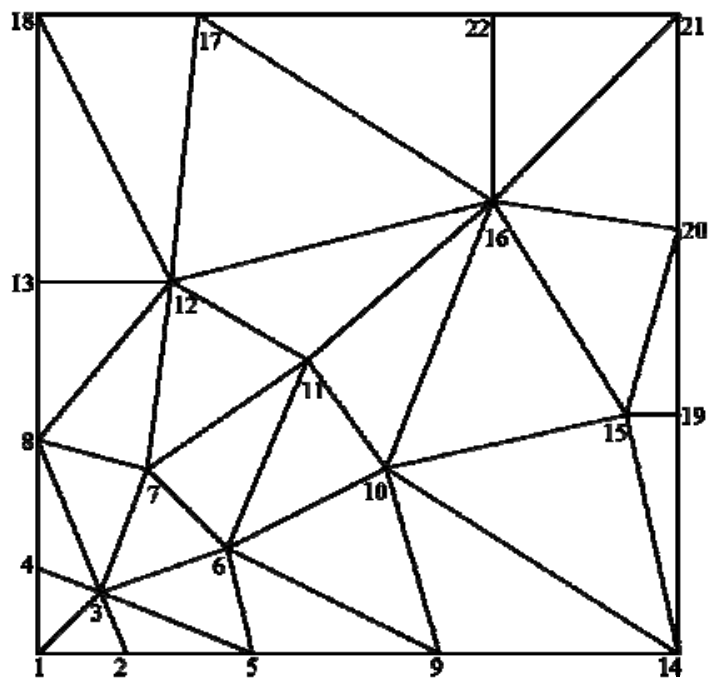

Fig. 2 Triangular grid.
The results of the comparison of the interlaced sweep method with such methods as: the sweep method for successive displacement along the grid lines, the modified sweep method, and the method of successive over-relaxation for two-dimensional area are shown in the table 1.

Similar results for the three-dimensional area are shown in the table 2 .

TABLE 1

THE RESULTS OF SOLVING THE CONTROL EXAMPLE IN TWODIMENSIONAL AREA

\begin{tabular}{|l|l|l|l|}
\hline Name of the method & $\begin{array}{l}\text { Coefficient } \\
\text { of } \\
\text { relaxation }\end{array}$ & $\begin{array}{l}\text { The } \\
\text { number of } \\
\text { iteration }\end{array}$ & error \\
\hline $\begin{array}{l}\text { Successive over- } \\
\text { relaxation }\end{array}$ & 1.77 & 98 & $0.28 \%$ \\
\hline $\begin{array}{l}\text { Sweep for } \\
\text { successive } \\
\text { displacement along } \\
\text { the lines }\end{array}$ & 1.0 & 368 & $0.06 \%$ \\
\hline $\begin{array}{l}\text { Modified sweep } \\
\text { method }\end{array}$ & 1.76 & 54 & $0.01 \%$ \\
\hline Interlaced sweep & 1.74 & 47 & $0.016 \%$ \\
\hline
\end{tabular}

TABLE 2

THE RESULTS OF SOLVING THE CONTROL EXAMPLE IN THREEDIMENSIONAL AREA

\begin{tabular}{|l|l|l|l|}
\hline Name of the method & $\begin{array}{l}\text { Coefficient } \\
\text { of } \\
\text { relaxation }\end{array}$ & $\begin{array}{l}\text { The } \\
\text { number of } \\
\text { iteration }\end{array}$ & error \\
\hline $\begin{array}{l}\text { Successive over- } \\
\text { relaxation }\end{array}$ & 1.81 & 95 & $0.006 \%$ \\
\hline $\begin{array}{l}\text { Sweep for } \\
\text { successive } \\
\text { displacement along } \\
\text { the lines }\end{array}$ & 1.0 & 416 & $0.05 \%$ \\
\hline $\begin{array}{l}\text { Modified sweep } \\
\text { method }\end{array}$ & 7.9 & 65 & $0.012 \%$ \\
\hline Interlaced sweep & 7.9 & 47 & $0.017 \%$ \\
\hline
\end{tabular}

\section{CONCLUSION}

The number of operations to be performed at each point of the grid when solving the problems by the methods listed in the table differs slightly, and the speeds of convergence differ in several times. The modification of the sweep method for sequential displacement along the grid lines has increased the speed of its convergence in 6 times, but the method of interlaced sweep turned out to be more effective. This proves the high efficiency of the interlaced sweep method.

By solving the problems by the finite element method, the nodes of touching of neighboring elements (Fig. 2) can be placed on curved lines, and the sweep on these lines can be performed observing the principle of interlaced sweep. By following this method, the neighboring lines should not belong to the same half-step. 
Aloizs Ratnieks, et al./ Environment. Technology. Resources, (2015), Volume III, 170-174

\section{REFERENCES}

[1] O. Zienkiewicz, R. L Taylor and D. D. Fox, The Finite Element Method for Solid and Structural Mechanics. ELSEVIER, 2011.
[2] William F. Ames, Numerical Methods For Partial Differential Equations. Academic Press Inc, 1992.

[3] В. Вазов, Дж. Форсайт. Разностные методы решения дифференииальных уравнений в частных производных. ИЛ, М., 1963. 